\title{
Oxidative Desulfurization of Diesel Fuel Using Three Kinds of Carboxylic Acids Supported by Iron(III) Chloride
}

\author{
Jalil H. Kareem* \\ Department of Petroleum Technology, Erbil Technology College, Erbil Polytechnic University, Erbil, Kurdistan Region, Iraq
}

\author{
${ }^{*}$ Corresponding author: \\ Jalil H. Kareem, Department \\ of Petroleum Technology, \\ Erbil Technology College, \\ Erbil Polytechnic University, \\ Erbil, Iraq. \\ E-mail: jalil.kareem@epu. \\ edu.iq
}

Received: 20 October 2020

Accepted: 31 January 2021

Published: 30 June 2021

\section{DOI}

10.25156/ptj.v11n1y2021. pp104-111

\section{A B S T R A C T}

Aqueous carboxylic acids (CA) are generally used as attractive catalytic extractants in the field of desulfurization of petroleum products. In the present study, a triple system consisting of CA-ferric chloride-hydrogen peroxide has been used for the removal of aromatic S-compounds from partially hydrotreated diesel by liquid - liquid oxidative-extraction. The influence of various operating parameters affecting the oxidative desulfurization was experimentally investigated. Formic acid ( $\mathrm{HCOOH}$ ), acetic acid $\left(\mathrm{CH}_{3} \mathrm{COOH}\right)$, and propanoic acid $\left(\mathrm{CH}_{3} \mathrm{CH}_{2} \mathrm{COOH}\right)$ as aqueous solutions with hydrogen peroxide and iron(III) chloride $\left(\mathrm{FeCl}_{3}\right)$ as oxidant and catalytic agent, respectively, were used. All experiments were carried out at $55^{\circ} \mathrm{C}$ with different oxidant to sulfur mole ratios $\left(\mathrm{nH}_{2} \mathrm{O}_{2} / \mathrm{nS}\right)(15-36), \mathrm{CA}$ to sulfur mole ratios $(\mathrm{nCA} / \mathrm{nS})(2-26)$, and oxidation reaction times $(5-25 \mathrm{~min})$. Within $25 \mathrm{~min}$ of the treatment, a maximum elimination of aromatic S-compounds of $65.1 \%$ was obtained when molar ratio of oxidant to sulfur $\left(\mathrm{nH}_{2} \mathrm{O}_{2} / \mathrm{nS}\right)$ was 36 and molar ratio of $\mathrm{CA}$ to sulfur $(\mathrm{nCA} / \mathrm{nS})$ was 26 . Surface tension calculations for the CAs demonstrated that the average void radius of the acids has a pronounced effect on the fitting of S-compounds from diesel fuel into acids and is important to sulfur removal. Further, increasing the desulfurization efficiency was also energetically affected in the presence of ferric halide. The obtention of the sulfur removal ability value was noticeably higher than was achieved by employing similar reaction conditions in the absence of $\mathrm{FeCl}_{3}$.

Keywords: Oxidation; Diesel fuel; Ferric chloride; Hydrogen peroxide; Pollution; Desulfurization

\section{INTRODUCTION}

Recently, the proportion of heavy low sulfur-containing oils in total petroleum production being treated and produced has increased drastically worldwide as the largest deposits of low and light sulfur hydrocarbons. The existence of heteroatomic molecules in petroleum feedstock not only effects the decreasing of the American Petroleum Institute (API) gravity value and poisoning catalytic convertors, but also reduces the quality of the resulting petroleum products. A significant part of the harmful materials of petroleum's components in the composition of fuels, such as sulfur and nitrogen, in which high-toxicity combustion products $\left(\mathrm{SO}_{\mathrm{x}}\right.$ and $\left.\mathrm{NO}_{\mathrm{x}}\right)$ are emitted into the atmosphere, thereby causing environmental pollution such as corrosion of construction work, harms agricultural products, impairs soil quality, and causes acid rain (Kobotaeva and Skorokhodova, 2020; Dehkordi et al., 2009). Diesel quality is of particular interest. Accordingly, removal of sulfur derivatives from petroleum has become an essential part of related industry processes. High sulfur content in Iraqi crude oil which causes environmental pollution is also the main reason for its removal in fuel products (Dehkordi et al., 2009). To allow the transition of the Iraqi oil refining industry to the production of fuels that meet European standards, reducing aromatic S-containing compounds in oil products to a minimum (around 10-15 ppm) is vital (Kareem et al., 2019).

In the industry, hydrodesulfurization (HDS) is currently the major trend in hydrocarbons purification with regard to the removal of sulfur impurities in their hydrotreating using solid catalysts. However, this operation has a relatively low efficiency in terms of the removal of polycyclic organosulfur species such as dibenzothiophenes and their alkyl derivatives. In addition, it requires harsh operating conditions such as high temperatures $\left(300-400^{\circ} \mathrm{C}\right)$ and high pressures (20-130 atm) which leads to very high capital and operating costs (Andevary et al., 2020; Grange, 1980).

Selecting catalysts with both good extractive ability and high catalytic activity have become vital to the superdesulfurization of petroleum products (McIntosh and Schram, 1977). Accordingly, many alternative non- 
HDS technologies such as oxidation (Gao et al., 2018), extraction (Abbott et al., 2017), biodesulfurization (Gray et al., 2003), and adsorption (Tian et al., 2014) have been suggested. However, the non-HDS results imply that all these technologies are insufficient and need further work to represent practical means of oil product desulfurization (Shu et al., 2020). However, among these alternative nonHDS methods, oxidative extractive desulfurization (ODS) is one of the more promising processes for developing diesel fuel, which can typically reduce the concentration of refractory polyaromatic sulfur hydrocarbons in the fuel under moderate reaction conditions and at low cost (Campos-Martin et al., 2004). One of the advantages of ODS processes is the possibility of their implementation at atmospheric pressure and low temperature. Another significant feature of ODS is that most of the refractory aromatic sulfur molecules, which probably cannot be removed in the HDS process, investigated high chemical reactivity for oxidation in the ODS operation. Further, this method is a non-hydrogen-consuming desulfurization process (Campos-Martin et al., 2004; De Filippis and Scarsella, 2003).

Typically, ODS operation consists of two steps: Oxidation and extraction. When the oxidation reaction of S-molecules takes place in the presence of an appropriate oxidizing agent containing an appropriate catalyst, aromatic S-compounds are converted to their corresponding polar sulfone and/or sulfoxide compounds (Pawelec et al., 2011). Then, polar sulfones are easily separated from fuel using a highly polar solvent. All of the substances noted in the ODS process have a major role in the applications of the research experiments investigated (Bhutto et al., 2016; Polikarpova et al., 2018). Several types of catalytic agents and oxidants have been studied for use in the ODS method. In the presence of a catalyst like ionic liquids ( $\mathrm{Lu}$ et al., 2007), Brønsted acids (Zhao et al., 2007), and deep eutectic solvents (DES) (Lü et al., 2015), hydrogen peroxide $\left(\mathrm{H}_{2} \mathrm{O}_{2}\right)$ as an active oxidant has seen widespread use in this important area of "green chemistry" due to having a high concentration of active oxygen, a desirable influence on the reactions, it is inexpensive, easy to synthesis and produces $\mathrm{H}_{2} \mathrm{O}$ as the only by-product (Zhao et al., 2007). $\mathrm{H}_{2} \mathrm{O}_{2}$, for the oxidation of aromatic sulfur compounds, is energetically reactive in aqueous carboxylic acid (CA), leading to situ formation of peroxy acid (Khodaei et al., 2017).

In addition, to improve the extraction efficiency of S-compounds in petroleum products, many researchers have used metal in the extraction procedure in ODS such as $\mathrm{Fe}(\mathrm{II}), \mathrm{Zn}, \mathrm{Mg}, \mathrm{Cu}, \mathrm{Sn}$, and $\mathrm{Co}$ to enhance sulfur elimination (Zhao et al., 2014). Based on oxidative liquidliquid extraction, separation is carried out after oxidation.
The extraction can be performed using acetonitrile as the solvent due to its low boiling point, high polarity, and high extraction effectiveness factor (Shiraishi et al., 1998; Deshpande et al., 2005).

The aim of the present work is to develop a more efficient extraction process using hydrogen peroxide-ferric chloride-CA systems to remove organosulfur compounds of partially hydrotreated diesel produced by North Oil Company (Kirkuk Refinery, Kirkuk, Iraq) with a sulfur mass content of $0.593 \%$. The influence of different operating parameters such as $\mathrm{H}_{2} \mathrm{O}_{2} / \mathrm{S}, \mathrm{FeCl}_{3}$, and the CA/S molar ratios on the sulfur removal was studied. The performances of the three types of acid (i.e. formic acid, acetic acid, and propanoic acid [PA]) were also examined in this regard.

\section{MATERIALS}

The substrate utilized as the object of this investigation was a partially hydrotreated diesel with a $0.593 \%$ total sulfur content as produced by North Oil Company (Kirkuk Refinery, Iraq). Table 1 shows the physical and chemical properties of this particular diesel. All chemical materials utilized in this study, including PA (98\%), acetic acid $(96 \%)$, acetonitrile (99 wt. \%), and hydrogen peroxide $(50 \% \mathrm{w} / \mathrm{w})$, were of analytical grade as purchased from Scharlau Chemie (Spain). Formic acid (>99\%) was supplied by Chem-Lab NV (Belgium). Ferric chloride solution $(60 \% \mathrm{w} / \mathrm{v})$ was provided by BDH (England). All chemicals were employed without further purification.

\section{METHODOLOGIES}

\section{Diesel Oxidation}

Oxidation-extraction experiments were performed in $40 \mathrm{~mL}$ tubes, containing $20 \mathrm{ml}$ of partially treated diesel. The reactor was placed in a constant temperature water bath on a hotplate coupled to a digital thermostat. For each

Table 1: Some basic physicochemical properties of diesel
used in this work
\begin{tabular}{|lcl}
\hline Characteristic & Amount & Test methods \\
\hline Initial boiling point (IBP) - ${ }^{\circ} \mathrm{C}$ & 180 & ASTM D7345 \\
Final boiling point (FBP) - ${ }^{\circ} \mathrm{C}$ & 346 & ASTM D7345 \\
Density @ $15^{\circ} \mathrm{C} \mathrm{-} \mathrm{g/ml}$ & 0.816 & ASTM 052 \\
Sulfur content - $\%$ & 0.593 & ASTM D4294 \\
Flash point - ${ }^{\circ} \mathrm{C}$ & 68 & ASTM D 6450 \\
Kinematic viscosity @ $40{ }^{\circ} \mathrm{C}-\mathrm{cSt}$ & 2.28 & ASTM D7042 \\
Cetane number & 51.7 & FTIR \\
Cetane index & 52.1 & ASTM D 976 \\
Water content - ppm & 41 & ASTM D6304 \\
Color & 0.8 & ASTM D 66045 \\
Pour point - ${ }^{\circ} \mathrm{C}$ & -14 & ASTM D 97 \\
Aromatic containing compounds - $\%$ & 28.3 & ASTM D1319 \\
\hline
\end{tabular}


experiment, an appropriate amount of each of the three CA and iron halide solution was added to the tube reactor with the diesel, and the mixture was agitated vigorously at $400 \mathrm{rpm}$. During the oxidation period, an appropriate amount of oxidant was added to the mixture, where this point in time was considered to represent the beginning reaction for experimental purposes. The reactions of the samples were performed for a certain amount of time and the temperature maintained at a constant $55 \pm 1{ }^{\circ} \mathrm{C}$. After the oxidation stage, the organic and aqueous layers were separated by simple decanting. The elimination of oxidized hydrocarbon in the aqueous phase was performed by washing the oxidized diesel with an equal volume of the deionized water. Thereafter, an equal volume of acetonitrile was introduced to the treated sample in a separating funnel which was shaking manually for about $5 \mathrm{~min}$ to remove the oxidized sulfur products. The extraction stage was repeated in triplicate. Ultimately, a sulfur analysis was obtained. Because there can be more readily regenerated, acetonitrile was used (Aghaei et al., 2020). Based on the system, practically, the single influence of aqueous $\mathrm{H}_{2} \mathrm{O}_{2}$ on a sulfur molar ratio $\left(\mathrm{H}_{2} \mathrm{O}_{2} / \mathrm{S}\right)$ that was varied between 15 and 37 and a CA to sulfur mole ratio (CA/S) from 2 to 26, and oxidation time of 5 to $25 \mathrm{~min}$, was examined. To obtain more accurate results each experiment was repeated 3 times, whereby the reproducibility of the replicated measurements could be seen from the error bars.

\section{Analytical Methods}

Diesel desulfurization was carried out using a triple system consisting of hydrogen peroxide (oxidant) and iron(III) chloride as a catalyst with formic acid, acetic acid, and PA as catalytic media with concentrations depending on total moles of sulfur in the feedstock. The sulfur content in the organic phase before and after oxidation has measured using X-ray fluorescence (Tanaka Scientific sulfur Meter Model RX-360SH, Japan) (Figure 1). According to ASTM D-4294, after bombarding a material (sulfur elements) in petroleum products with high-energy X-rays or gamma rays, secondary or fluorescent rays are emitted from it that has been excited. The experimental approach is based on the energy dispersive $\mathrm{X}$-ray fluorescence technique.

The minimum applicable sulfur detection in this technique is at least $10 \mathrm{ppm}$. The total amounts of sulfur removed with these compounds were calculated according to the equation (1):

$$
\text { Desulfurization efficiency }(\%)=\left(1-\frac{S_{i}}{S_{f}}\right) \times 100
$$

where $S_{i}$ and $S_{f}$ are sulfur content of the initial diesel, and the sulfur content of the oxidized diesel, respectively. To

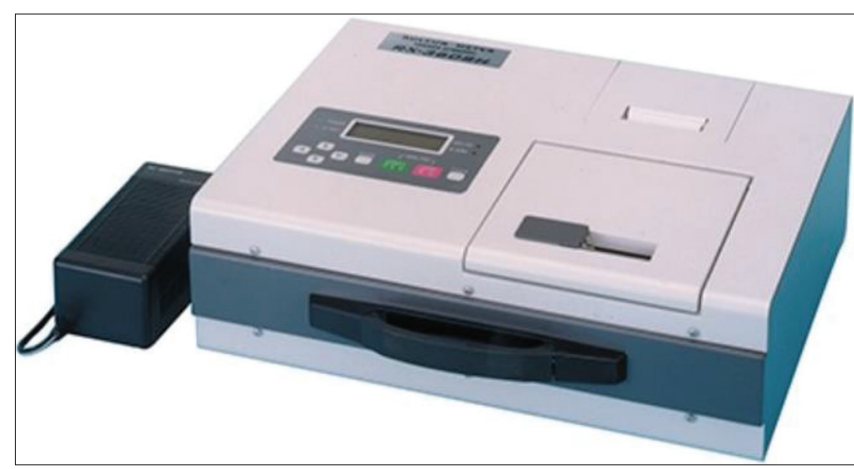

Figure 1: X-ray fluorescence device (Tanaka Scientific Sulfur Meter Model RX-360SH) that has used to measure total sulfur contents in diesel

obtain more accurate results, each experiment was repeated 3 times and the error bars for all the data were given.

A Krüss Tensiometer K9 model K9MK1 was employed to measure CA surface tensions. A glass dish containing the acid was surrounded by a water jacket connected to a thermostat to control the temperature of the acid sample. Surface tensions were recorded wherein a Pt-Ir alloy plate was immersed in the acid surface layer.

\section{RESULTS AND DISCUSSION}

Table 1 summarizes the physicochemical properties of diesel produced by Kirkuk Refinery. As can be seen, the boiling point range for the diesel used in the extraction is $180-346^{\circ} \mathrm{C}$. It has been analyzed that the basic sulfur species of fuel products in the boiling range of diesel are almost alkylbenzothiophenes and alkyldibenzothiophenes (Ma et al., 1994). To investigate the effects of several operating parameters on the oxidative sulfur reduction process, various mixtures of immiscible solutions (CALewis acid-Fe(III) halide) in diesel fuel were made up for oxidation with $\mathrm{H}_{2} \mathrm{O}_{2}$ and which played a triple role in the process of sulfur removal. All oxidation reactions were carried out with varying molar ratios of $\mathrm{H}_{2} \mathrm{O}_{2} / \mathrm{S}$ and CA/S at a constant $55^{\circ} \mathrm{C}$. Moreover, in comparison with simple extraction to reduce sulfur from diesel, ODS of diesel using ferric halides was carried out to investigate the importance of the catalyst-oxidation stage before the extraction stage in the final stage of desulfurization.

Ferric chloride has a pronounced effect on the extraction of organo S-compounds from oil products. Dharaskar et al., 2015, noted that due to the specific interaction between the $\mathrm{Fe}^{3+}$ cation and aromatic sulfur compounds, up to $59 \%$ of sulfur species could be extracted into the Lewis acidic $\mathrm{FeCl}_{3}$-based (Bmim)Cl phase. Similarly, Zhang et al., 2009, extracted around $96 \%$ of thiophenic compounds from model fuel into anhydrous ferric chloride-based $(\mathrm{Bmim}) \mathrm{BF}_{4}$ 
ionic liquid. In the current study, four different iron(III) concentrations were used under otherwise identical reaction conditions to examine the effects of the amount of cation on the oxidative reaction properties (Table 2). $\mathrm{FeCl}_{3}$ increased the extraction ability to about $4-7 \%$ of the ODS process.

What is interesting about the data in this table is that the sulfur removal gradually increased from 14 to $21 \%$ and 15.5 to $21 \%$ in $\mathrm{FA}$ and $\mathrm{AA}$, respectively, as $\left[\mathrm{Fe}^{3+}\right]$ was varied from 3.7 to $11.1 \mathrm{mmol} / \mathrm{L}$. However, the sulfur removal dropped as the $\mathrm{Fe}^{\mathrm{III}}$ Lewis acid concentration was changed from 11.1 to $14.8 \mathrm{mmol} / \mathrm{L}$ with sulfur removals of $21 \%$ to $20 \%$ and $21 \%$ to $19 \%$ in FA and AA, respectively. The reason behind the decrease in desulfurization efficiency was possibly due to fact that using high concentrations of $\mathrm{FeCl}_{3}$ enhanced the decomposition of $\mathrm{H}_{2} \mathrm{O}_{2}$. Based on the above result, I have used $11.1 \mathrm{mmol} / \mathrm{L}$ in the ODS process.

\section{Effect of Mole ratio Of CA/S on Reactions}

As mentioned in the literature review, during the oxidation stage, various non-aliphatic sulfur compounds were oxidized to their corresponding sulfoxide and sulfones. CA might be the best solvents that act as extractants in the oxidation desulfurization through the formation of percarboxylic acids, which catalyze and give their oxygen atoms to the sulfur-containing species present in diesel (Ali et al., 2006). Percarboxylic acid is produced in situ from CA and the oxidant $\left(\mathrm{H}_{2} \mathrm{O}_{2}\right)$. In the ODS process, it was found that an increase in extractant dosage has a pronounced effect on its extraction power, and therefore the sulfur removal efficiency will significantly increase (Lü et al., 2013).

Based on this, the ODS reaction can be improved by increasing the mass of the $\mathrm{R}-\mathrm{COOH}$ in the reaction. In addition, CA is well known as a good extractant for the simultaneous extraction of the oxidized sulfur-based molecules in the reaction medium compared to aldehydes and ketones. To investigate the influence of stoichiometric of $\mathrm{nCA} / \mathrm{nS}$ on the performance of oxidation reactions, the oxidation reactions for $\mathrm{AA}$ and $\mathrm{FA}$ at $55 \pm 1{ }^{\circ} \mathrm{C}$, with stoichiometries of $\mathrm{nCA} / \mathrm{nS}=2,8,14,20$, and 26 , a mole ratio of $\mathrm{H}_{2} \mathrm{O}_{2} / \mathrm{S}=15$, time of reaction $=25 \mathrm{~min}$ were

Table 2: Influence of the concentration of $\mathrm{FeCl}_{3}$ on the removal of S-compounds

\begin{tabular}{lcc}
\hline$\left[\mathrm{FeCl}_{3}\right] / \mathrm{mmol} / \mathrm{L}$ & \multicolumn{2}{c}{ S-removal $(\mathrm{ppm})$} \\
\cline { 2 - 3 } & At FA & At AA \\
\hline 3.7 & $819.7 \pm 7.8$ & $914.7 \pm 10.4$ \\
7.4 & $1080 \pm 8.2$ & $1123.4 \pm 9.4$ \\
11.1 & $1230 \pm 4.7$ & $1251.6 \pm 8.5$ \\
14.8 & $1203.3 \pm 8.4$ & $1133.5 \pm 4.5$ \\
\hline
\end{tabular}

Experimental conditions: $\mathrm{T}=55^{\circ} \mathrm{C}, \mathrm{t}=25 \mathrm{~min}, \mathrm{nCA} / \mathrm{nS}=4, \mathrm{nH}_{2} \mathrm{O}_{2} / \mathrm{nS}=7$, $\mathrm{S}_{\mathrm{i}}=5930 \mathrm{ppm}$ and mixing speed $=400 \mathrm{rpm}$ carried out. Whereas Xu et al., 2018, used a PA-based DES for deep desulfurization, due to its complete solubility in diesel fuel, PA could not be utilized as an aqueous catalytic medium for the ODS system in this work, as shown in Figure 2. To demonstrate the miscibility of PA in choline chloride as DES, I have prepared a eutectic solvent according to their experimental method and mixed this with diesel and n-heptane; however, the PA-based DES completely dissolved in both non-polar phases.

With an increasing number of methylene groups, the hydrophobic interaction increases, as analyzed by Yamamoto and Nishi, 1990. There is a significant difference in the hydrophobicity of methyl, ethyl, and propyl groups which is probably enough once diarized to make the difference. Therefore, only AA and FA were used as aqueous extractants. The percentage sulfur removal versus $\mathrm{nCA} / \mathrm{nS}$ is shown in Figure 3, where the stock weight concentration of sulfur molecules was $0.593 \%$.

In the present experiment, it was shown that the sulfur elimination increases with increasing $\mathrm{CA} / \mathrm{S}$ mole ratio. When the mole ratio of $\mathrm{nCA} / \mathrm{nS}$ in $\mathrm{FA}$ and $\mathrm{AA}$ increased from 2 to 26, the sulfur removal increases from 36 to $55 \%$ and 38 to $61.8 \%$, respectively. In the reaction medium, an increasing CA concentration is the consequence of the expected increment in percarboxylic acid concentration that leads to enhanced in situ extraction of oxidized S-compounds in the lower layer of the aqueous reaction medium. Extraction and catalytic oxidation in the CA/ $\mathrm{FeCl}_{3}$ solution clearly removes the sulfur from the diesel. Moreover, the capability of ODS for S-removal in AA is higher than in FA as shown in Figure 3.

Recent evidence suggests that surface tension is an indicator and an important aspect of understanding the required energy to increase the surface area and form a

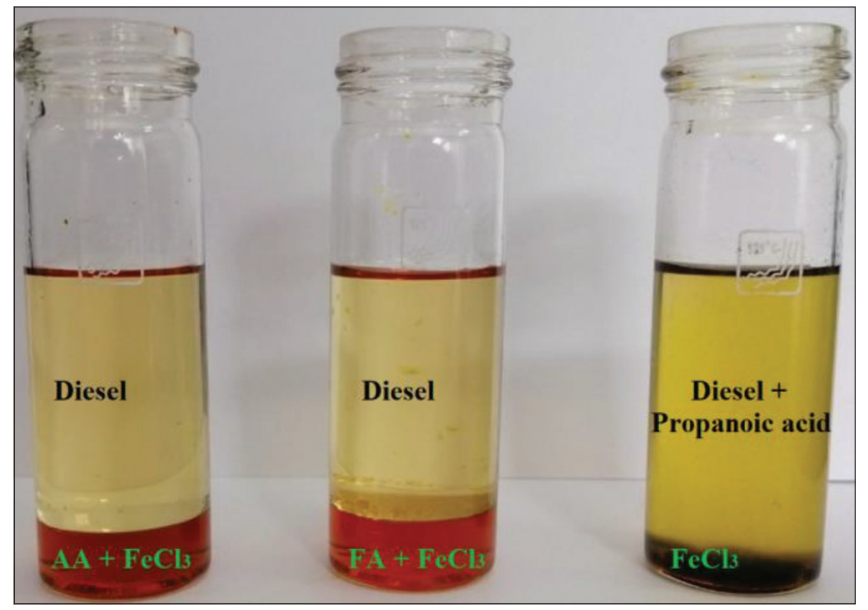

Figure 2: Forming two phases in extraction by AA and FA, and one phase by propanoic acid after adding diesel 
hole in the fluid for the S-compounds to fit into (Chandler, 2005, Abbott et al., 2017). High surface tension prevents the extracted liquid from forming an emulsion due to mechanical excitation, and hence reduced extraction efficiency. Any decrease in the cohesive forces at the interface result in low surface tension, hence increasing liquid solubilization (Abbott et al., 2017). Using Krüss tensiometer, the surface tensions $(\gamma)$ at $55^{\circ} \mathrm{C}$ were measured for AA $\left(23.4 \mathrm{mN} \cdot \mathrm{m}^{1}\right)$ and $\mathrm{FA}\left(33.5 \mathrm{mN} \cdot \mathrm{m}^{1}\right)$ to calculate the average radius of the holes $\left(\mathrm{r}_{\mathrm{H}}\right)$ of the extractants using equation 2 (Abbott et al., 2007):

$$
\mathrm{r}_{\mathbf{H}}=\sqrt{ }(3.5 K T / 4 \pi \gamma)
$$

where $T$ is the temperature in Kelvin and $K$ is the Boltzmann constant.

Average void radius $r_{H}(\AA)$ of $\mathrm{AA}$ and $\mathrm{FA}$ extractants were 23.2 and 19.1 respectively. It is cleared that AA has lower surface tension than the FA which directly proportional with the capability of the extraction. It can be deduced that the energy required to make a hole in the $\mathrm{AA}$ is lower than in the FA due to the bigger average hole size, which is ultimately the controlling factor limiting the transportation of aromatic S-species from the non-polar diesel phase into the $\mathrm{CA}$.

\section{Effect of $\mathrm{H}_{2} \mathrm{O}_{2}$ /S Mass Ratio on ODS}

In the R-COOH- $\mathrm{H}_{2} \mathrm{O}_{2}$ extraction-oxidation sulfur elimination system with oxidant (i.e. $\mathrm{H}_{2} \mathrm{O}_{2}$ ), the dosage of $\mathrm{H}_{2} \mathrm{O}_{2}$ directly influences the amount of peroxycarboxyl produced in the oxidation process, and thus has a direct effect on the desulfurization efficiency (Zhang et al., 2011). The influence of the $\mathrm{H}_{2} \mathrm{O}_{2}$ as a unique oxidizing agent to sulfur mole ratio on the ODS of the aromatic sulfur compounds present in diesel was investigated at $55^{\circ} \mathrm{C}$. Based on the stoichiometry of the reaction, two moles of oxidant should be consumed per mole of sulfone product (equation 3) (Campos-Martin et al., 2004).

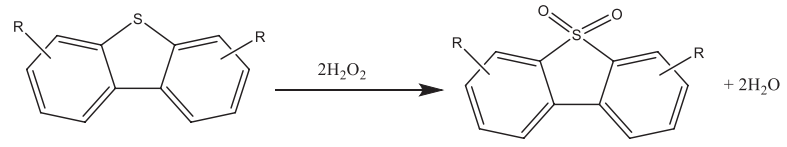

$\mathrm{H}_{2} \mathrm{O}_{2} / \mathrm{S}$ molar ratios of $15,22,29$, and 36 were selected, and the removal of heterocyclic sulfur compounds over 25 min was plotted, as per Figure 4.

It can be has seen in the figure that the removal of aromatic sulfur species gradually increases from $55.8 \%$ to $65 \%$ and $51 \%$ to $59.1 \%$ when using AA and FA, respectively, when the mole ratio of $\mathrm{H}_{2} \mathrm{O}_{2} / \mathrm{S}$ increased from 15 to 36. Whereas the oxidizing agent is usually employed in excess of the stoichiometric ratio in ODS, in the present findings the

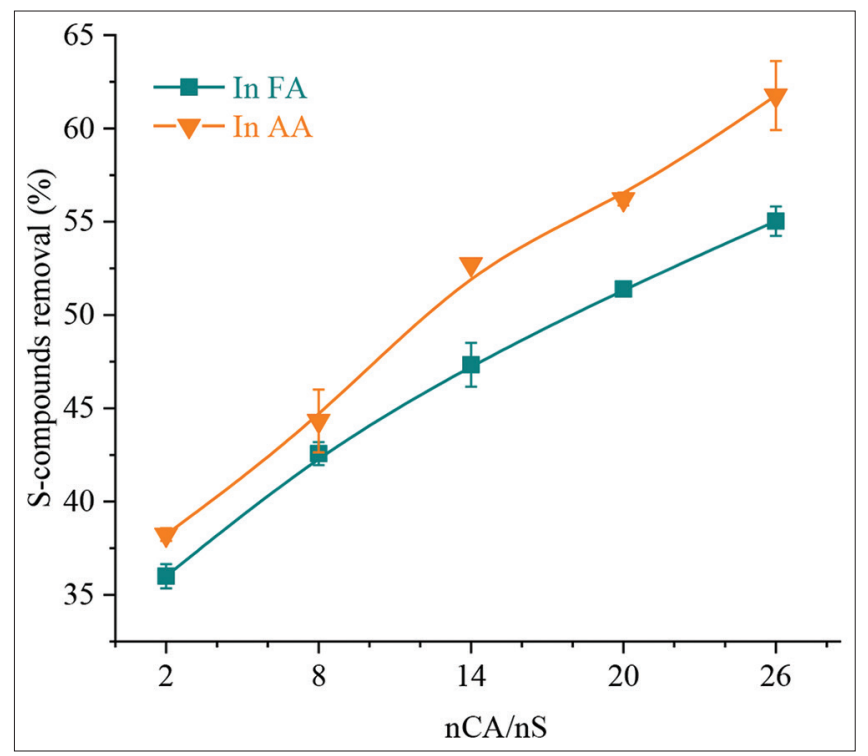

Figure 3: Effect of the molar ratio of carboxylic acids/S on sulfur removal. Reaction conditions: $\mathrm{H}_{2} \mathrm{O}_{2}-\mathrm{FeCl}_{3}$-carboxylic acid system; $\mathrm{T}=55^{\circ} \mathrm{C} ; \mathrm{Si}=0.593 \% ;\left[\mathrm{FeCl}_{3}\right]=11.1 \mathrm{mmol} / \mathrm{L}, \mathrm{nH}_{2} \mathrm{O}_{2} / \mathrm{nS}=15$ (i.e., for oxidation of $20 \mathrm{ml}$ of diesel, $2.58 \mathrm{ml} \mathrm{H}_{2} \mathrm{O}_{2}$ and $0.345,1.38,2.42$, 3.46 , and $4.5 \mathrm{ml}$ of acetic acid and 0.23, 0.91, 1.6, 2.3, and $2.96 \mathrm{ml}$ of formic acid for nAA/nS or nFA/nS equal to $2,8,14,20$, and 26 , were used, respectively)

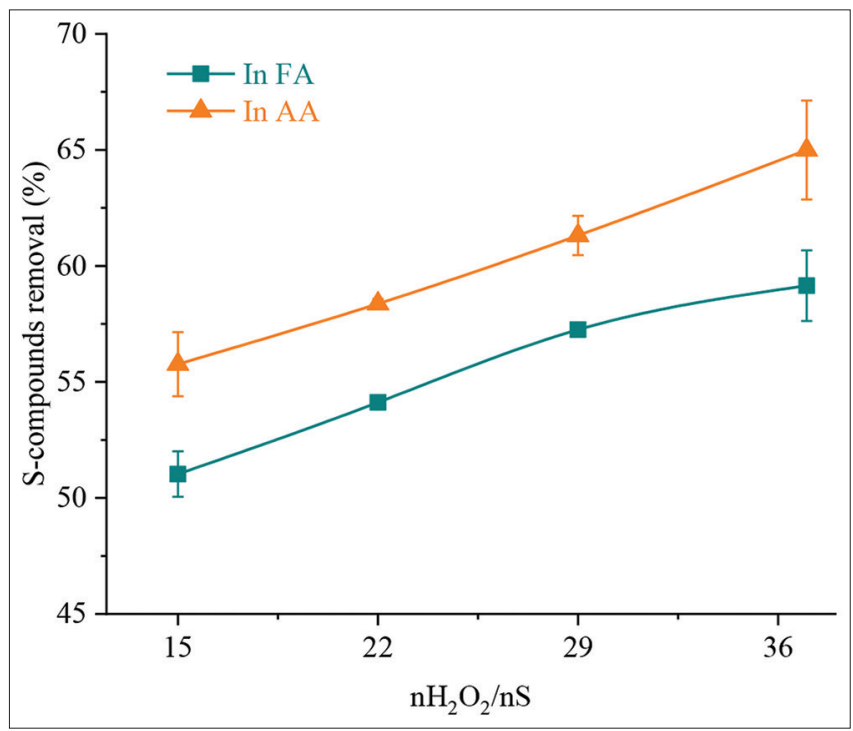

Figure 4: Sulfur removal as a function of $\mathrm{H} 2 \mathrm{O} 2 / \mathrm{S}$ mole ratio. Reaction conditions: $\mathrm{H}_{2} \mathrm{O}_{2}-\mathrm{FeCl}_{3}$ - carboxylic acid system; $\mathrm{T}=55^{\circ} \mathrm{C} ; \mathrm{Si}=0.593 \% ;\left[\mathrm{FeCl}_{3}\right]=11.1 \mathrm{mmol} / \mathrm{L}, \mathrm{nCA} / \mathrm{nS}=26$ (i.e., for oxidation of $20 \mathrm{ml}$ diesel, $4.5 \mathrm{ml}$ acetic acid, $2.96 \mathrm{ml}$ formic acid, and 2.58, 3.77, 4.98, and $6.2 \mathrm{ml} \mathrm{H}_{2} \mathrm{O}_{2}$ and for $\mathrm{nH}_{2} \mathrm{O}_{2} / \mathrm{nS}$ ratios of 15 , 22,29 , and 36 , were used, respectively)

maximum removal capability appeared to be up to $65 \%$ of the sulfur compounds in diesel, which was expected and essentially unsurprising. The first possible reason for just $65 \%$ removal of S-compounds is shown in Table 1, namely, that $28.3 \%$ of diesel is made up of aromatic species, some of which will probably be polyromantic compounds such as 
phenanthrenes that prevent sulfur extraction from fuel, as highlighted by Xiao et al., 2012. Due to high concentrations of aromatic compounds in diesel, the cetane number is reduced to 51.7 and negatively impacts the fuel's quality. The second possible reason is due to the lack of efficient mass transfer between the aqueous layer and diesel phase reaction systems (Feng, 2010). While $55^{\circ} \mathrm{C}$ as a reaction temperature has been used in this work, oxygen and water are products of thermal decomposition has been reported by Hiroki and LaVerne, 2005.

Another problem is with the consumption of oxidant by the nitrogen-containing compounds' co-oxidation reactions in the diesel, which seriously complicate matters. These reactions are undesirable because of the consequent high oxygen consumption and because all $\mathrm{N}$-compounds appear to inhibit ODS reactions of aromatic S-species, as reported by Caero et al., 2006. Another possible reason for the efficiency of the ODS of used diesel in this study is that the addition of peroxide to petroleum products results in epoxidation of olefins in tandem with the oxidation of sulfur compounds making this method ineffective, as investigated by Ali et al., 2006. In addition, finding an appropriate extractant to efficiently adsorb sulfur molecules rather than aromatic hydrocarbons and olefins is a particular challenge to the ODS process. Abro et al., reported that choosing unsuitable extractants has a pronounced effect on the undesired parallel extraction of S-free olefins or aromatics from the fuel liquid with the intended sulfones (Abro et al., 2014). A maximum excess of oxidant was $6 \mathrm{ml}$ versus $20 \mathrm{ml}$ diesel when $\mathrm{nH}_{2} \mathrm{O}_{2} / \mathrm{nS}$ was 36 , because if a very high excess of hydrogen peroxide had been used in this study, the operation would be no longer representative of any industrial application. From the abovementioned reasons, the ODS process remains a matter of controversy.

\section{Effect of Reaction Time on Sulfur Elimination}

The efficiency of removing organic S-compounds from the partially hydrotreated diesel as a function of various reaction times $(5,10,15,20$, and $25 \mathrm{~min})$ at a $55^{\circ} \mathrm{C}$ reaction temperature and at $400 \mathrm{rpm}$ was examined (Figure 5). According to the van't Hoff law, increasing temperature up to $70^{\circ} \mathrm{C}$ in extraction reactions results in a decline in sulfur removal capability. Thus, the extraction process becomes exothermic and S-compounds start to repartition from the extractant phase to the diesel phase (Rahma et al., 2017). In addition, a high reaction temperature results in catalyst deactivation and $\mathrm{H}_{2} \mathrm{O}_{2}$ decomposition (Tian et al., 2015). Lü et al. found that at room temperature, the ODS is relatively low compared to a higher temperatures due to reduced $\mathrm{H}_{2} \mathrm{O}_{2}$ reactivity (Lü et al., 2015). Therefore, $55^{\circ} \mathrm{C}$ was utilized as the reaction temperature.

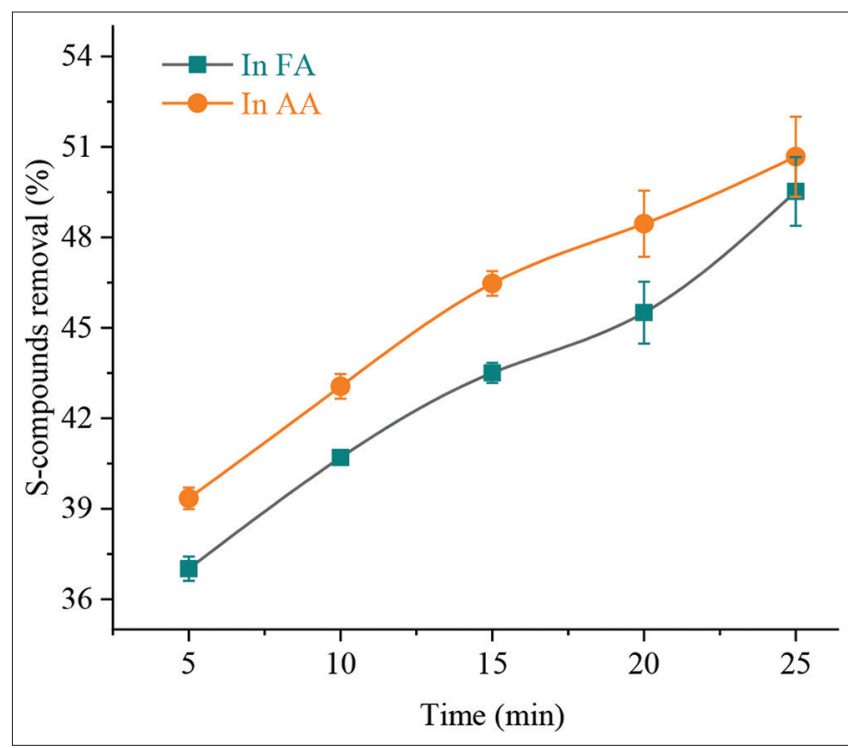

Figure 5: Oxidative desulfurization from diesel as a function of time. Reaction conditions: $\mathrm{H}_{2} \mathrm{O}_{2}-\mathrm{FeCl}_{3}$-carboxylic acid system; $\mathrm{T}=55^{\circ} \mathrm{C} ; \mathrm{Si}=0.593 \% ;\left[\mathrm{FeCl}_{3}\right]=11.1 \mathrm{mmol} / \mathrm{L}, \mathrm{nCA} / \mathrm{nS}=20$ (i.e., for oxidation of $20 \mathrm{ml}$ of diesel, $3.46 \mathrm{ml}$ of acetic acid, $2.96 \mathrm{ml}$ formic acid, and $5 \mathrm{ml}$ of $\mathrm{H}_{2} \mathrm{O}_{2}$ were used, respectively)

It is shown from Figure 5 that the extractive desulfurization in both AA and FA improved from 39.4 to $50.7 \%$ and 37 to $49.5 \%$, respectively, as the reaction time increased from 5 to 25 min. The reaction rates in AA and FA are clearly rapid, as the S-removal after $5 \mathrm{~min}$ had reached 39.4 and $37 \%$, respectively. These results indicate that oxidantsupported ferric chloride is a key factor affecting S-removal. The aqueous solution of $\mathrm{H}_{2} \mathrm{O}_{2}-\mathrm{FeCl}_{3}-\mathrm{R}-\mathrm{CO}_{2} \mathrm{H}$ system is mostly caught in the bottom phase of two immiscible biphasic systems. Thus, it can be operatively reutilized for sulfur removal in new feeds, which can be considered as an important feature of the ODS system.

\section{CONCLUSIONS}

In this study, the ODS of partially hydrotreated diesel using the triple system of $\mathrm{H}_{2} \mathrm{O}_{2}$-ferric chloride-CA (formic acid, acetic acid, or PA) was studied experimentally. The influences of different operating parameters such as reaction time, $\mathrm{FeCl}_{3}$ concentration, and the $\mathrm{nH}_{2} \mathrm{O}_{2} / \mathrm{nS}$, and $\mathrm{nCA} / \mathrm{nS}$ ratio on the performance of the ODS operation were examined.

Due to the difference in hydrophobicity between formic acid, acetic acid, and PA, the latter dissolved fully in diesel and thus could not be used as an aqueous extractant. There is a good correlation between the sulfur compound removal and the energy required to make a hole in the FA and AA. It was shown that AA (lower surface tension) showed better extraction performance than FA (higher surface tension), 
because the average void radius in AA $(23.2 \AA)$ is bigger than that of FA $(19.1 \AA)$.

An $\mathrm{nH}_{2} \mathrm{O}_{2} / \mathrm{nS}$ molar ratio of 36 gave allowed for the greatest removal of sulfur compounds at $55^{\circ} \mathrm{C}$. The extraction of sulfur species in the desulfurization process of partially hydrotreated diesel in the absence of oxidation is not in any way an efficient method.

Increasing the molar ratio of $\mathrm{nCA} / \mathrm{nS}$ in reaction medium results in an increase in S-removal due to the associated increase in percarboxylic acid concentration, which enhances in situ extraction of oxidized sulfur compounds. Maximum sulfur-based compound removal observed in the current study was $65.1 \%$. This was due to the large amounts of aromatic-containing compounds in the diesel which negatively affected its quality and reduces its cetane number from a more normal range to 51.7.

\section{ACKNOWLEDGMENTS}

The author would like to thank Erbil Polytechnic University for funding this work, and further thanks goes to the Polytechnic Research Centre and Koya Technical Institute in EPU for their facilities and help. The authors also thank Dr Mark Watkins from the University of Leicester for proofreading this manuscript.

\section{REFERENCES}

Abbott, A. P., A. Y. Al-Murshedi, O. A. Alshammari, R. C. Harris, J. H. Kareem, I. B. Qader and K. Ryder. 2017. Thermodynamics of phase transfer for polar molecules from alkanes to deep eutectic solvents. Fluid Phase Equil. 448: 99-104.

Abbott, A. P., R. C. Harris and K. S. Ryder. 2007. Application of hole theory to define ionic liquids by their transport properties. J. Phys. Chem. B. 111: 4910-4913.

Abro, R., A. A. Abdeltawab, S. S. Al-Deyab, G. Yu, A. B. Qazi, S. Gao and $X$. Chen. 2014. A review of extractive desulfurization of fuel oils using ionic liquids. Res. Adv. 4: 35302-35317.

Aghaei, A., S. Shahhosseini and M. A. Sobati. 2020. Regeneration of different extractive solvents for the oxidative desulfurization process: An experimental investigation. Proc. Saf. Environ. Protect. 139: 191-200.

Ali, M. F., A. Al-Malki, B. El-Ali, G. Martinie and M. N. Siddiqui. 2006. Deep desulphurization of gasoline and diesel fuels using nonhydrogen consuming techniques. Fuel. 85: 1354-1363.

Andevary, H. H., A. Akbari, Z. Rajabi and M. Omidkhah. 2020. Towards a room temperature oxidative desulfurization of refractory compounds over 1-octyl-3-methylimidazolium tetrachloroferrates/silica gel: The beneficial effects of immobilization. Proc. Saf. Environ. Protect. 136: 343-352.

Bhutto, A. W., R. Abro, S. Gao, T. Abbas, X. Chen and G. Yu. 2016. Oxidative desulfurization of fuel oils using ionic liquids: A review. J. Taiwan Inst. Chem. Eng. 62: 84-97.

Caero, L. C., F. Jorge, A. Navarro and A. Gutiérrez-Alejandre. 2006. Oxidative desulfurization of synthetic diesel using supported catalysts: Part II. Effect of oxidant and nitrogen-compounds on extraction-oxidation process. Catal. Today. 116: 562-568.

Campos-Martin, J., M. Capel-Sanchez and J. Fierro. 2004. Highly efficient deep desulfurization of fuels by chemical oxidation. Green Chem. 6: 557-562.

Chandler, D. 2005. Interfaces and the driving force of hydrophobic assembly. Nature. 437: 640-647.

De Filippis, P. and M. Scarsella. 2003. Oxidative desulfurization: Oxidation reactivity of sulfur compounds in different organic matrixes. Energy Fuels. 17: 1452-1455.

Dehkordi, A. M., M. A. Sobati and M. A. Nazem. 2009. Oxidative desulfurization of non-hydrotreated kerosene using hydrogen peroxide and acetic acid. Chine. J. Chem. Eng. 17: 869-874.

Deshpande, A., A. Bassi and A. Prakash. 2005. Ultrasound-assisted, base-catalyzed oxidation of 4, 6-dimethyldibenzothiophene in a biphasic diesel-acetonitrile system. Energy Fuels. 19: 28-34.

Dharaskar, S. A., K. L. Wasewar, M. N. Varma and D. Z. Shende. 2015. FeCl 3 based imidazolium ionic liquids as novel solvents for extractive-oxidative desulfurization of liquid fuels. J. Solut. Chem. 44: 652-668.

Feng, M. 2010. Review on recent patents in sulfur removal from liquid fuels by oxidative desulfurization (ODS) process. Rec. Patents Chem. Eng. 3: 30-37.

Gao, J., S. Zhu, Y. Dai, C. Xiong, C. Li, W. Yang and X. Jiang. 2018. Performance and mechanism for extractive desulfurization of fuel oil using modified polyethylene glycol. Fuel. 233: 704-713.

Grange, P. 1980. Catalytic hydrodesulfurization. Catal. Rev. Sci. 21: 135-181.

Gray, K. A., G. T. Mrachko and C. H. Squires. 2003. Biodesulfurization of fossil fuels. Curr. Opin. Microbiol. 6: 229-235.

Hiroki, A. and J. A. Laverne. 2005. Decomposition of hydrogen peroxide at water-ceramic oxide interfaces. J. Phys. Chem. B, 109: 3364-3370.

Kareem, J. H., A. P. Abbott and K. Ryder. 2019. Shifting desulfurization equilibria in ionic liquid-oil mixtures. Energy Fuels. 33: 11061113.

Khodaei, B., M. A. Sobati and S. Shahhosseini. 2017. Rapid oxidation of dibenzothiophene in model fuel under ultrasound irradiation. Monatshefte Für Chem. Chem. Monthly. 148: 387-396.

Kobotaeva, N. and T. Skorokhodova. 2020. Ionic Liquids as Extractants of Diesel Sulfur Compounds. Petroleum Chem. 60: 762-767.

Lü, H., P. Li, C. Deng, W. Ren, S. Wang, P. Liu and H. Zhang. 2015. Deep catalytic oxidative desulfurization (ODS) of dibenzothiophene (DBT) with oxalate-based deep eutectic solvents (DESs). Chem. Commun. 51: 10703-10706.

Lü, H., W. Ren, H. Wang, Y. Wang, W. Chen and Z. Suo. 2013. Deep desulfurization of diesel by ionic liquid extraction coupled with catalytic oxidation using an Anderson-type catalyst [(C4H9) $4 \mathrm{~N}]$ 4NiMo6O24H6. Appl. Catal. A General. 453: 376-382.

Lu, L., S. Cheng, J. Gao, G. Gao and M. Y. He. 2007. Deep oxidative desulfurization of fuels catalyzed by ionic liquid in the presence of H2O2. Energy Fuels. 21: 383-384.

Ma, X., K. Sakanishi and I. Mochida. 1994. Hydrodesulfurization reactivities of various sulfur compounds in diesel fuel. Ind. Eng. Chem. Res. 33: 218-222.

Mcintosh, J. M. and C. K. Schram. 1977. Reductive desulfurization using tributyltin hydride. Can. J. Chem. 55: 3755-3757.

Pawelec, B., R. M. Navarro, J. M. Campos-Martin and J. L. Fierro. 2011. Retracted article: Towards near zero-sulfur liquid fuels: A perspective review. Catal. Sci. Technol. 1: 23-42. 
Polikarpova, P., A. Akopyan, A. Shigapova, A. Glotov, A. Anisimov and E. Karakhanov. 2018. Oxidative desulfurization of fuels using heterogeneous catalysts based on MCM-41. Energy Fuels. 32: 10898-10903.

Rahma, W. S. A., F. S. Mjalli, T. Al-Wahaibi and A. A. Al-Hashmi. 2017. Polymeric-based deep eutectic solvents for effective extractive desulfurization of liquid fuel at ambient conditions. Chem. Eng. Res. Design. 12000: 271-283.

Shiraishi, Y., T. Hirai and I. Komasawa. 1998. A deep desulfurization process for light oil by photochemical reaction in an organic two-phase liquid-liquid extraction system. Ind. Eng. Chem. Res. 37: 203-211.

Shu, C., F. Lai, F. Zhu and D. Luo. 2020. Optimal extractive and reductive desulfurization process using sodium borohydride in situ generated via sodium metaborate electroreduction in ionic liquid. Chem. Eng. Proc. Proc. Intens. 150: 107869.

Tian, F., Q. Shen, Z. Fu, Y. Wu and C. Jia. 2014. Enhanced adsorption desulfurization performance over hierarchically structured zeolite Y. Fuel Proc. Technol. 128: 176-182.

Tian, Y., Y. Yao, Y. Zhi, L. Yan and S. Lu. 2015. Combined extractionoxidation system for oxidative desulfurization (ODS) of a model fuel. Energy Fuels. 29: 618-625.

Xiao, J., C. Song, X. Ma and Z. Li. 2012. Effects of aromatics, diesel additives, nitrogen compounds, and moisture on adsorptive desulfurization of diesel fuel over activated carbon. Ind. Eng. Chem. Res. 51: 3436-3443.

Xu, H., D. Zhang, F. Wu, X. Wei and J. Zhang. 2018. Deep desulfurization of fuels with cobalt chloride-choline chloride/ polyethylene glycol metal deep eutectic solvents. Fuel. 225: 104-110.

Yamamoto, K. and N. Nishi. 1990. Hydrophobic hydration and hydrophobic interaction of carboxylic acids in aqueous solution: Mass spectrometric analysis of liquid fragments isolated as clusters. J. Am. Chem. Soc. 112: 549-558.

Zhang, C., W. Feng, X.Y. Pan and X.Q. Liu. 2011. Study of extractionoxidation desulfurization of model oil by acidic ionic liquid. J. Fuel Chem. 39: 689-693.

Zhang, J., W. Zhu, H. Li, W. Jiang, Y. Jiang, W. Huang and Y. Yan. 2009. Deep oxidative desulfurization of fuels by Fenton-like reagent in ionic liquids. Green Chem. 11: 1801-1807.

Zhao, D., J. Wang and E. Zhou. 2007. Oxidative desulfurization of diesel fuel using a Brønsted acid room temperature ionic liquid in the presence of H2O2. Green Chem. 9: 1219-1222.

Zhao, Y., X. Wen, T. Guo and J. Zhou. 2014. Desulfurization and denitrogenation from flue gas using Fenton reagent. Fuel Proc. Technol. 128: 54-60. 